Pacific Journal of Mathematics

DEFORMATION OF SUBMANIFOLDS OF REAL PROJECTIVE 


\title{
DEFORMATION OF SUBMANIFOLDS OF REAL PROJECTIVE SPACE
}

\author{
KICHOON YANG
}

\begin{abstract}
We prove that two surfaces in $\mathbf{R P}^{3}$ are projective deformations of each other (in the sense of $E$. Cartan) if and only if their induced projective structures are equivalent with respect to the Frenet framing. This result gives a projective generalization of molding surfaces.
\end{abstract}

0. Introduction. Equivalence problems of induced structures on submanifolds of homogeneous spaces are closely related to so called $G$-deformation problems. In the case of surfaces in $\mathbf{R P}^{3} \cong \operatorname{PGL}(4 ; \mathbf{R}) / G_{0}$ the projective deformation ( $\operatorname{PGL}(4 ; \mathbf{R})$-deformation) problem has been investigated by $\mathrm{E}$. Cartan (cf. [2]). In this paper we establish a relationship between the notion of projective deformation of surfaces and the notion of equivalence of induced projective structures on surfaces. Our main result is that two surfaces in $\mathbf{R P}^{3}$ are projective deformations of each other (in the sense of E. Cartan) if and only if their induced projective structures are equivalent with respect to the Frenet framing. (Theorem 5.3)

To better understand the geometric content of our result consider the analogous situation from classical surface theory. Take two surfaces in $\mathbf{R}^{3} \cong \mathbf{E}(3) / O(3)$. Then the $\mathbf{E}(3)$-deformation problem is just the equivalence problem of induced Riemannian structures (i.e., the problem of local isometry). However, $\mathbf{E}(3)$-deformations in general do not preserve the Frenet frames (of Euclidean geometry) whereas, as we shall show, PGL $(4 ; \mathbf{R})$-deformations do preserve the Frenet frames (of projective geometry). Requiring that an $\mathbf{E}$ (3)-deformation should preserve the Frenet frames is equivalent to requiring that the corresponding local isometry preserves the lines of curvature, and the solutions to this latter problem are given by molding surfaces. (See $[1], \S 5$.) Our result may be thought of as a projective analog of molding surfaces.

Several features distinguish the projective differential geometry from the Euclidean differential geometry:

(i) A projective structure is a $G$-structure of degree two, i.e., it is a subbundle of the bundle of quadratic frames, and 
(ii) an induced projective structure on a submanifold is not uniquely determined. In fact, it depends upon a choice of normal frame field.

The first order deformation problem becomes trivial (Theorem 4.4) reflecting the fact that a projective structure is a $G$-structure of degree two.

We now explain the organization of our paper.

The principal tools to be used are the notions of Pfaffian systems and their prolongations. In $\S 1$ the notion of prolongation is defined and applying the technique inductively an upper bound for the number of successive prolongations required to obtain singular integrals of the system is computed. This section constitutes a general method for attacking over-determined Pfaffian systems in two independent variables.

In $§ 2$ we discuss moving frames on submanifolds of $\mathbf{R P}^{n}$. In particular, the construction of Frenet frames on surfaces in $\mathbf{R} \mathbf{P}^{3}$ are given.

In $\$ 3$ we give a brief exposition on the general theory of induced projective structures and their equivalences.

In $\$ 4$ we derive exterior systems describing projective deformations of submanifolds in $\mathbf{R P}^{n}$. The system describing first order deformations is seen to be involutive and the system describing second order deformations is seen to be over-determined in general.

In $\$ 5$ we consider surfaces in $\mathbf{R P}^{3}$. We show that for generic surfaces the exterior system describing second order deformations is over-determined. Applying the technique of prolongation it is shown that second order deformations take the Frenet frames to the Frenet frames: This gives the theorem that the second order deformation implies the equivalence of induced projective structures with respect to the Frenet frames. The converse implication is also proven. We complete the process of successive prolongations arriving at the integrability condition of $\mathrm{E}$. Cartan (see [2], p. 288). This solves the problem of determining when non-trivial second order deformations exist: It also gives an upper bound on the dimension of the parameter space of the surface that are second order deformations of a given surface.

We shall work within the category of smooth maps and objects, except where we apply the Cartan-Kähler theorem and draw positive conclusions in which case we must assume real analyticity. Such assumptions will be explicitly made.

Finally, we acknowledge a great debt to E. Cartan's work. In particular, this paper grew out of an attempt to understand his paper [2]. The author is also grateful to Gary Jensen for several helpful discussions on E. Cartan's work and $G$-deformation problems in general. 
1. Prolongations of Pfaffian systems. A Pfaffian system on a manifold $M$ of dimension $m$ can be thought of as a subbundle of the linear frame bundle $L(M)$ defined by the equations

$$
\Sigma:\left\{\theta^{\alpha}=0: \theta^{\alpha} \in T^{*} M, i=1,2, \ldots, s\right\},
$$

where we assume that 1 -forms $\left(\theta^{\alpha}\right)$ are linearly independent.

Fix a $p$-dimensional submanifold $S_{0} \stackrel{i_{0}}{\rightarrow} M$. We introduce the following index convention which will be used in this section:

$$
\begin{aligned}
& 1 \leq i, j, k, \cdots \leq p, \\
& 1 \leq \alpha, \beta, \gamma, \cdots \leq s, \\
& 1 \leq a, b, c, \cdots \leq m-(p+s) .
\end{aligned}
$$

Let $\left(\omega^{i}, \omega^{a}, \omega^{\alpha}\right)$ be a coframe field on $M$ so that $i_{0}^{*}\left(\omega^{1} \wedge \omega^{2} \wedge \cdots \wedge \omega^{p}\right)$ $\neq 0$. We will be interested in finding $p$-dimensional integral submanifolds $S \stackrel{i}{\rightarrow} M$ so that $i_{*} T S$ projects onto $i_{0^{*}} T S_{0}$. That being so, we may as well assume that $\omega^{1} \wedge \omega^{2} \wedge \cdots \wedge \omega^{p} \wedge \theta^{1} \wedge \cdots \wedge \theta^{s} \neq 0$, since otherwise such integral submanifolds clearly do not exist. Possibly rechoosing $\left(\omega^{a}, \omega^{\alpha}\right)$ we assume that $\left(\omega^{i}, \omega^{a}, \theta^{\alpha}\right)$ form a coframe field on $M$.

We differentiate $\left(\theta^{\alpha}\right)$ and, suppressing the terms containing $\left(\theta^{\alpha}\right)$, obtain the following.

$$
d \theta^{\alpha} \equiv A_{a i}^{\alpha} \omega^{a} \wedge \omega^{l}+\frac{1}{2} B_{l j}^{\alpha} \omega^{l} \wedge \omega^{j}+\frac{1}{2} C_{a b}^{\alpha} \omega^{a} \wedge \omega^{b} \quad \text { (modulo } \Sigma \text { ), }
$$
where $A_{a l}^{\alpha}, B_{l j}^{\alpha}=-B_{j i}^{\alpha}, C_{a b}^{\alpha}=-C_{b a}^{\alpha}$ are functions on $M$.

Definition 1.1. We say that the system is in normal form if $\left(C_{a b}^{\alpha}\right) \equiv 0$.

The significance of the system in normal form is that in such a case Cartan's involutivity criterion reduces the existence question of generic integral submanifolds to linear algebra.

Let $\left(e_{i}, e_{a}, e_{\alpha}\right)$ be the frame field on $M$ dual to $\left(\omega^{l}, \omega^{a}, \theta^{\alpha}\right)$. We will choose a local coordinate system on the Grassman bundle of $p$-planes $G_{p}(M) \rightarrow M$, about the $p$-dimensional plane field defined by $e_{1} \wedge e_{2} \wedge$ $\cdots \wedge e_{p}$, arising from the choice $\left(\omega^{l}, \omega^{a}, \theta^{\alpha}\right)$. A $p$-plane $E \in G_{p}(M)$ in the neighborhood defined by $\omega^{1} \wedge \omega^{2} \wedge \cdots \wedge \omega^{p} \neq 0$ is represented by an $n \times p$ matrix,

$$
\left[\begin{array}{l}
\left(\delta_{j}^{l}\right) \\
\left(l_{j}^{a}\right) \\
\left(l_{j}^{\alpha}\right)
\end{array}\right] .
$$

That is to say, $E$ is given by a decomposable $p$-vector $v_{1} \wedge v_{2} \wedge \cdots \wedge v_{p}$, where $v_{j}=e_{J}+l_{J}^{a} e_{a}+l_{j}^{\alpha} e_{\alpha}$. We take $\left(l_{J}^{a}, l_{J}^{\alpha}\right)$ as the standard fibre coordinates on $G_{p}(M)$. 
We now express the condition that $\left(\theta^{\theta}\right)$ and $\left(d \theta^{\alpha}\right)$ vanish on $E \in$ $G_{p}(M)$ in terms of the standard coordinates on $G_{p}(M)$. (Such elements $E \in G_{p}(M)$ are called integral elements; observe that they are possible tangent spaces of $p$-dimensional integral submanifolds.) On such an $E \in G_{p}(M)$, we have $\theta^{\alpha}=l_{j}^{\alpha} \omega^{j}=0, \omega^{a}=l_{j}^{a} \omega^{j}, d \theta^{\theta}=0$. Using (1.2) and substituting, we obtain,

$$
\begin{gathered}
F_{i}^{\alpha}: l_{i}^{\alpha}=0, \\
F_{i j}^{\alpha}: A_{a j}^{\alpha} l_{i}^{a}-A_{a i}^{\alpha} l_{j}^{a}+B_{i j}^{\alpha}+C_{a b}^{\alpha}\left(l_{i}^{a} l_{j}^{b}-l_{i}^{b} l_{j}^{a}\right)=0 .
\end{gathered}
$$

Observe that the variety in $G_{p}(M)$ defined by $\left(F_{i}^{\alpha}\right) \equiv\left(F_{i j}^{\alpha}\right) \equiv 0$ may be empty. We then say that the system $\Sigma$ is incompatible. We also note that if $\Sigma$ is in normal form then equations (1.3) and (1.4) define a system of inhomogeneous linear equations, and hence the variety in question is irreducible.

We now define the prolongation of $\Sigma$.

DEFINITION 1.2. The prolongation of $\Sigma$, denoted by $\Sigma^{\prime}$ is the exterior differential system on $G_{p}(M)$ given by

$$
\begin{aligned}
& \Sigma^{\prime}=\Sigma^{(0)} \cup \Sigma^{(1)} \cup \Sigma^{\prime(2)}, \text { where } \\
& \Sigma^{\prime(0)}:\left\{F_{i}^{\alpha}=0, F_{i j}^{\alpha}=0\right\}, \\
& \Sigma^{\prime(1)}:\left\{d F_{i}^{\alpha}=0, d F_{i j}^{\alpha}=0, \theta^{\alpha}=0, \omega^{a}-l_{j}^{a} \omega^{j}=0\right\}, \\
& \Sigma^{\prime(2)}: d \Sigma^{\prime(1)} .
\end{aligned}
$$

Here, and elsewhere, we write $\theta^{\alpha}$ in place of $\pi^{*} \theta^{\alpha}$, where $\pi: G_{p}(M)$ $\rightarrow M$, etc.

Restricting to the variety $V \subset G_{p}(M)$ defined by $\Sigma^{\prime(0)}$ we obtain a Pfaffian system on $V$, which we denote by $\hat{\Sigma}^{\prime}$. Observe that $\hat{\Sigma}^{\prime}$ is always in normal form.

We now consider the case of $s$ equations in $(s-1)$-unknowns ("slightly over-determined system") with two independent variables.

Let $M$ be a $(2 s+1)$-dimensional manifold. On $M$ we are given the Pfaffian system

$$
\Sigma:\left\{\theta^{\alpha}=0, \alpha=1,2, \ldots, s\right\} .
$$

Choose 1 -forms $\left(\omega^{i}, \omega^{a}\right)$ on $M$ so that $\left(\omega^{i}, \omega^{a}, \theta^{\alpha}\right)$ form a coframe field. We will be interested in integral surfaces on which $\omega^{1} \wedge \omega^{2} \neq 0$. Note that our index convention becomes $1 \leq i, j \leq 2,1 \leq a, b \leq s-1,1 \leq \alpha$, $\beta \leq s$.

Assuming that $\Sigma$ is in normal form we write down $d \Sigma$, modulo $\Sigma$ as usual,

$$
d \Sigma:\left\{d \theta^{\alpha} \equiv A_{a i}^{\alpha} \omega^{a} \wedge \omega^{i}+B^{\alpha} \omega^{1} \wedge \omega^{2}=0\right\}
$$


We now think of $\left(A_{a i}^{\alpha}\right)$ as an $s$ by $2(s-1)$ matrix $\left(A_{A}^{\alpha}\right)$ by setting $A=(i-1)(s-1)+a$. We assume that the rank of $\left(A_{A}^{\alpha}\right)$ is maximal, i.e., equal to $s$. (If not, then further assuming that the system does not possess any "characteristic" (i.e., trivial) solutions, it can be shown that the system becomes involutive possibly with compatibility conditions.) Suffice it to say that then the system is not involutive.

We prolong the system. Keeping earlier notations we obtain:

$$
\begin{gathered}
\Sigma^{\prime(0)}:\left\{F_{i}^{\alpha}=0, F_{i j}^{\alpha}=0\right\}, \text { where } \\
F_{l}^{\alpha}=l_{i}^{\alpha}=0, \\
F_{i j}^{\alpha}=A_{a j}^{\alpha} l_{t}^{a}-A_{a i}^{\alpha} l_{j}^{a}+B^{\alpha}=0 .
\end{gathered}
$$

Letting $B=(2-i)(s-1)+a$, we think of $\left(l_{i}^{a}\right)$ as a vector $\left(l^{B}\right) \in$ $\mathbf{R}^{2(s-1)}$. Also letting $\left(v^{B}\right)={ }^{\mathrm{t}}\left(l^{1}, l^{2}, \ldots, l^{s-1},-l^{s}, \ldots,-l^{2(s-1)}\right)$ equations (1.8) become

$$
A_{B}^{\alpha} v^{B}=B^{\alpha} .
$$

The solutions of (1.9) form an (s-2)-dimensional affine space. It follows that $V \subset G_{2}(M)$ defined by $\Sigma^{(0)}$ is an irreducible variety over $M$ of fibre dimension $(s-2)$. Let $t=\left(t^{1}, t^{2}, \ldots, t^{s-2}\right)$ be a fibre coordinate system in $V$. Then $\hat{\Sigma}^{\prime}$ on $V$ is given by

$$
\hat{\Sigma}^{\prime}:\left\{\theta^{\alpha}=0, \omega^{a}-\hat{l}_{j}^{a}(t) \omega^{j}=0\right\},
$$

where $\left(\hat{l}_{j}^{a}(t)\right)$ denote the solutions of (1.9).

Observing that $d \theta^{\alpha} \equiv 0\left(\bmod \hat{\Sigma}^{\prime}\right)$ we compute that

$$
d \hat{\Sigma}^{\prime}:\left\{\theta^{\alpha}=d \omega^{a}-\hat{l}_{j}^{a}(t) d \omega^{j}-d \hat{l}_{j}^{a}(t) \omega^{j}=0, a=1,2, \ldots, s-1\right\} .
$$

Rewriting the equations in $d \hat{\Sigma}^{\prime}$, we obtain

$$
d \hat{\Sigma}^{\prime}:\left\{\theta^{\alpha} \equiv a_{\varepsilon j}^{a} d t^{\varepsilon} \wedge \omega^{j}+b^{a} \omega^{1} \wedge \omega^{2}=0\right\},
$$

where $\left(a_{\varepsilon j}^{a}\right)$ and $\left(b^{a}\right)$ are functions on $V$ and $1 \leq \varepsilon \leq s-2$. We see that $d \hat{\Sigma}^{\prime}$ contains $(s-1)$ equations $\left(\theta^{\alpha}\right)=0$ and $(s-2)$ unknowns $\left(d t^{\varepsilon}\right)$. Now applying the technique of prolongation successively we obtain the

TheOREM 1.3. Over-determined normal Pfaffian system with two independent variables in $s$ equations and $(s-1)$ unknowns require at the most $(s-1)$ successive prolongations in order for the system to be either incompatible or involutive.

2. Higher order moving frames. We construct higher order moving frames on submanifolds of $\mathbf{R P}^{n}$. For a description of the general theory dealing with the problems of higher order contacts and frames of submanifolds of homogeneous spaces we refer to [5]. 
For the rest of the paper we adhere to the following index convention:

$$
\begin{aligned}
1 & \leq \alpha, \beta, \gamma, \cdots \leq n, \\
1 & \leq i, j, k, \cdots \leq p, \\
p+1 & \leq a, b, c, \cdots \leq n .
\end{aligned}
$$

$\mathbf{R P}^{n}$ is the real projective space of dimension $n$ which we think of as lines in $\mathbf{R}^{n+1}$. PGL $(n+1 ; \mathbf{R})$, the group of projective transformations of $\mathbf{R P}{ }^{n}$, is $\mathrm{GL}(n+1 ; \mathbf{R})$ modulo its center. Letting the $x$-axis to be the origin of $\mathbf{R P}^{n}, \mathbf{R P}^{n}$ is realized as a homogeneous space $\operatorname{PGL}(n+1 ; \mathbf{R}) / G_{0}$, where $G_{0}$ is the isotropy subgroup at the origin.

If $\left(X_{B}^{A}\right)_{0 \leq A, B \leq n} \in \mathrm{GL}(n+1 ; \mathbf{R})$ with $X_{0}^{0} \neq 0$, then set $a^{\alpha}=X_{0}^{\alpha} / X_{0}^{0}$, $a_{\beta}^{\alpha}=X_{\beta}^{\alpha} / X_{0}^{0}, a_{\beta}=X_{\beta}^{0} / X_{0}^{0}$. We take $\left(a^{\alpha}, a_{\beta}^{\alpha}, a_{\beta}\right)$ as a local coordinate system in the neighborhood of the identity of $\operatorname{PGL}(n+1 ; \mathbf{R})$ defined by $X_{0}^{0} \neq 0$. Then $\left(a^{\alpha}\right) \equiv 0$ defines $G_{0}$.

The Lie algebra $\mathscr{P} \mathscr{G} \mathscr{L}(n+1 ; \mathbf{R})$ is the vector space direct sum $\mathscr{P} \mathscr{G} \mathscr{L}(n+1 ; \mathbf{R})=\mathscr{M}_{0} \oplus \mathscr{G L}(n ; \mathbf{R}) \oplus \mathscr{M}_{0}^{*}$ with the obvious bracket operations, where $\mathscr{M}_{0} \cong \mathbf{R}^{n}$. Let $\Omega=\left(\Omega^{\alpha}, \Omega_{\beta}^{\alpha}, \Omega_{\beta}\right)$ be the Maurer-Cartan form of $\operatorname{PGL}(n+1 ; \mathbf{R})$. It is $\mathscr{M}_{0} \oplus \mathscr{G} \mathscr{L}(n ; \mathbf{R}) \oplus \mathscr{M}_{0}^{*}$-valued and its components are the left-invariant 1 -forms on $\operatorname{PGL}(n+1 ; \mathbf{R})$ which coincide with $d a^{\alpha}, d a_{\beta}^{\alpha}, d a_{\beta}$ at the identity. We record the structure equations:

$$
\left\{\begin{array}{l}
d \Omega^{\alpha}=-\Omega_{\gamma}^{\alpha} \wedge \Omega^{\gamma}, \\
d \Omega_{\beta}^{\alpha}=-\Omega_{\gamma}^{\alpha} \wedge \Omega_{\beta}^{\gamma}-\Omega^{\alpha} \wedge \Omega_{\beta}+\delta_{\beta}^{\alpha} \Omega_{\gamma} \wedge \Omega^{\gamma}, \\
d \Omega_{\beta}=-\Omega_{\gamma} \wedge \Omega_{\beta}^{\gamma} .
\end{array}\right.
$$

Let $f: S^{p} \hookrightarrow \mathbf{R P}^{n}$ be a $p$-dimensional submanifold given locally as a graph $\left(x^{i}\right) \mapsto\left(x^{i}, f^{a}(x)\right)$, where $x=\left(x^{i}\right)$. Let $L\left(\mathbf{R P}^{n}\right)$ denote the bundle of linear frames over $\mathbf{R} \mathbf{P}^{n}$. We choose the coordinates on $L\left(\mathbf{R P}^{n}\right)$ so that the natural projection $\operatorname{PGL}(n+1 ; \mathbf{R}) \rightarrow L\left(\mathbf{R P}^{n}\right)$ is given by $\left(a^{\alpha}, a_{\beta}^{\alpha}, a_{\beta}\right) \mapsto\left(a^{\alpha}, a_{\beta}^{\alpha}\right) \equiv\left(a^{\alpha}, e_{\beta}\right)$. Let $L_{0}$ denote the pull-back bundle $f^{-1} \operatorname{PGL}(n+1 ; \mathbf{R})$.

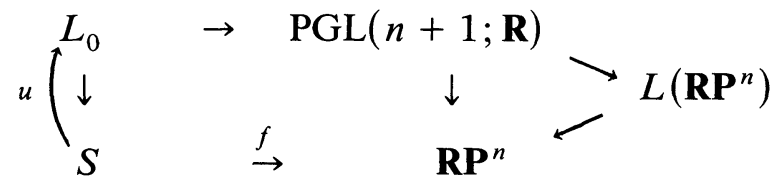

We call a section $u$ : $S \rightarrow L_{0}$ a zeroth order moving frame field along $f$. We see that a zeroth order moving frame field gives a local representation of the derivative map,

$$
d f=\phi^{\alpha} \otimes e_{\alpha}=u^{*} \Omega^{\alpha} \otimes e_{\alpha} \in \Gamma\left(T^{*}(S) \otimes f^{-1} T\left(\mathbf{R P}^{n}\right)\right) .
$$


Consider $u_{0}: S \rightarrow L_{0}$ given by

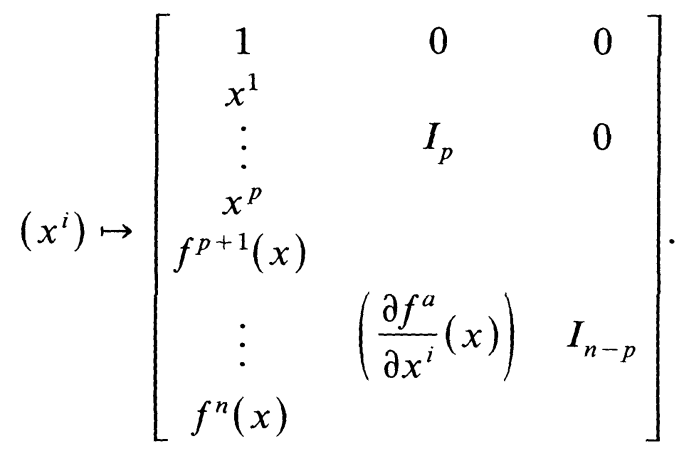

Then

$$
u_{0}^{*} \Omega^{a}=\left(u_{0}^{-1} d u_{0}\right)_{0}^{a}=-\frac{\partial f^{a}}{\partial x^{k}} d x^{k}+d f^{a}=0 .
$$

Hence $u_{0}$ normalizes $d f$ in the sense that

$$
d f=\phi^{1} \otimes e_{1} \oplus \cdots \oplus \phi^{p} \otimes e_{p},
$$

where $\left(\phi^{i}=u_{0}^{*} \Omega^{i}\right)$ form a coframe field on $S$.

Suppose we have another zeroth order moving frame field $u: S \rightarrow L_{0}$. Then $u=u_{0} \cdot g$, where $g: S \rightarrow G_{0}$ is some smooth map. Consider the group action of $G_{0}$ on $\mathscr{M}_{0}=\mathbf{R}^{n}$ defined by the equation $u^{*}\left(\Omega^{\alpha}\right)=$ $\left(u_{0} \cdot g\right)^{*}\left(\Omega^{\alpha}\right)$. Using the fact that $\left(u_{0} \cdot g\right)^{*}\left(\Omega^{\alpha}\right)=$ the $\mathscr{M}_{0}$-component of $\operatorname{Ad}\left(g^{-1}\right) u_{0}^{*} \Omega$, we compute that: if $g\left(a_{\beta}^{\alpha}, a_{\beta}\right)=\left(A, a_{\beta}\right) \in G_{0}$ and $v \in \mathbf{R}^{n}$, then the action is given by $v \mapsto A v$. The obvious isotropy subgroup for this action is $G_{1}<G_{0}$, where

(2.3) $G_{1}=\left\{\left[\begin{array}{ccc}1 & \left(a_{j}\right) & \left(\eta_{b}\right) \\ 0 & \left(a_{j}^{i}\right) & \left(\eta_{b}^{i}\right) \\ 0 & 0 & \left(b_{b}^{a}\right)\end{array}\right]: \begin{array}{l}\left(a_{j}^{i}\right) \in \mathrm{GL}(p: \mathbf{R}), \\ \left(a_{j}\right) \in \mathbf{R}^{p},{ }^{\mathrm{t}}\left(\eta_{b}\right) \in \mathbf{R}^{n-p}, \\ \left(\eta_{b}^{i}\right) \in \mathbf{R}^{p \times(n-p)},\left(b_{b}^{a}\right) \in \mathrm{GL}(n-p ; \mathbf{R})\end{array}\right\}$

Definition 2.1. The bundle of first order moving frames of $f$ is $L_{1}=\left\{u_{0} \cdot G_{1}\right\}$, where $u_{0}$ is given by (2.1).

$L_{1}$ is a $G_{1}$-principal bundle over $S$ and any section $u: S \rightarrow L_{1}$ is called a first order moving frame field along $f$. We emphasize that any first order moving frame field along $f$ normalizes $d f$ in the sense of (2.2).

On $L_{1}, \Omega^{a}=0$ for $p+1 \leq a \leq n$. Differentiating both sides of the equation and using the structure equations we obtain $\Omega_{i}^{a} \wedge \Omega^{i}=0$. By Cartan's lemma, it follows that

$$
\Omega_{J}^{a}=A_{j k}^{a} \Omega^{k}
$$

where $A_{j k}^{a}=A_{k J}^{a}$ are functions on $L_{1}$. 
Though the methods in what follows are quite general, for the remainder of this section we restrict ourselves to the case of surfaces in $\mathbf{R P}^{3}$ to ease computational difficulties and to simplify exposition. Note that our index convention becomes $1 \leq i, j, k, \cdots \leq 2,1 \leq \alpha, \beta, \gamma, \cdots$ $\leq 3$, and $a, b, c, \cdots=3$.

(2.4) becomes

$$
\left\{\begin{array}{l}
\Omega_{l}^{3}=a \Omega^{1}+b \Omega^{2}, \\
\Omega_{2}^{3}=b \Omega^{1}+c \Omega^{2},
\end{array}\right.
$$

where $a, b, c$, are functions on $L_{1}$.

Let

$$
E_{4}=\left|\begin{array}{llll}
0 & 0 & 0 & 0 \\
0 & 0 & 0 & 0 \\
0 & 0 & 0 & 0 \\
0 & 1 & 0 & 0
\end{array}\right| \text { and } E_{5}=\left|\begin{array}{cccc}
0 & 0 & 0 & 0 \\
0 & 0 & 0 & 0 \\
0 & 0 & 0 & 0 \\
0 & 0 & 1 & 0
\end{array}\right| .
$$

Then we have a vector space direct sum decomposition $\mathscr{G}_{0}=\mathscr{G}_{1} \oplus \mathscr{M}_{1}$, where $\mathscr{G}_{0}$ and $\mathscr{G}_{1}$ are Lie algebras for $G_{0}$ and $G_{1}$ respectively, and $\mathscr{M}_{1}=\operatorname{span}\left\{E_{4}, E_{5}\right\}$. We write $\left(\Omega_{i}^{3}\right)=\Omega_{l}^{3} \otimes E_{4} \oplus \Omega_{2}^{3} \otimes E_{5}=$ the $\mathscr{M}_{1^{-}}$ component of $\Omega$. Fix a first order moving frame field $\hat{u}: S \rightarrow L_{1}$. Write $\hat{u}^{*} \Omega_{i}^{3}=\hat{x}_{i k} \hat{u}^{*} \Omega^{k}$ for some functions $\hat{x}_{i k}$ on $S$. Suppose $u: S \rightarrow L_{1}$ is given by $u=\hat{u} \cdot g$, for a smooth map $g: S \rightarrow G_{1}$. Let $x_{i k}$ be functions on $S$ so that $u^{*} \Omega_{i}^{3}=x_{i k} u^{*} \Omega^{k}$. We will compute the action of $G_{1}$ on $\left(\hat{x}_{i k}\right)$. Observe that $u^{*}\left(\left(\Omega^{\alpha}\right) \oplus\left(\Omega_{i}^{3}\right)\right)=(\hat{u} \cdot g)^{*}\left(\left(\Omega^{\alpha}\right) \oplus\left(\Omega_{i}^{3}\right)\right)=$ the $\mathscr{M}_{0} \oplus \mathscr{M}_{1}$-component of $\operatorname{Ad}\left(g^{-1}\right) \hat{u}^{*} \Omega$. Some matrix multiplications show that

$$
X=g(\hat{X})=\frac{1}{b}{ }^{\mathrm{t}} A \hat{X} A,
$$

where

$$
g=\left[\begin{array}{cccc}
1 & \left(a_{j}\right) & \eta^{0} \\
0 & & & \\
& A & & \left(\eta^{i}\right) \\
0 & & & \\
0 & 0 & 0 & b
\end{array}\right] \in G_{1}, \quad X=\left(x_{j k}\right), \quad \hat{X}=\left(\hat{x}_{j k}\right) .
$$

There are four orbits of the above action represented by

$$
\left(\begin{array}{ll}
0 & 0 \\
0 & 0
\end{array}\right),\left(\begin{array}{ll}
1 & 0 \\
0 & 0
\end{array}\right),\left(\begin{array}{ll}
1 & 0 \\
0 & 1
\end{array}\right),\left(\begin{array}{ll}
0 & 1 \\
1 & 0
\end{array}\right)
$$

Assuming that $f$ is of constant orbit type, we call the first orbit type degenerate type, and the rest are called parabolic type, elliptic type, and hyperbolic type, respectively. 
We give a description of the bundle of second order moving frames, denoted by $L_{2}$, as follows.

Given a first order moving frame field $u: S \rightarrow L_{1}$ we write $u^{*} \Omega_{i}^{3}=$ $x_{i k} u^{*} \Omega^{k}\left(x_{i k}=x_{k i}\right.$ functions on $\left.S\right)$ as before. Let $\mathscr{S}_{2}$ denote the vector space of all real $2 \times 2$ symmetric matrices. We define $\Phi: L_{1} \rightarrow \mathscr{S}_{2}$ by the following commutative diagram.

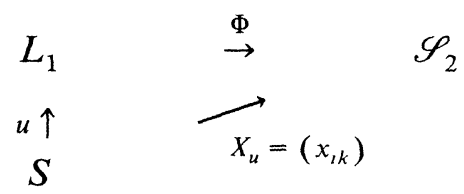

$L_{2}$ is then defined to be

$$
\Phi^{-1}\left\{\left(\begin{array}{ll}
0 & 0 \\
0 & 0
\end{array}\right)\right\}, \quad \Phi^{-1}\left\{\left(\begin{array}{ll}
1 & 0 \\
0 & 0
\end{array}\right)\right\}, \quad \Phi^{-1}\left\{\left(\begin{array}{ll}
1 & 0 \\
0 & 1
\end{array}\right)\right\} \quad \text { or } \quad \Phi^{-1}\left\{\left(\begin{array}{ll}
0 & 1 \\
1 & 0
\end{array}\right)\right\}
$$

depending upon the orbit type. $L_{2}$ is a $G_{2}$-principle bundle over $S$, where $G_{2}$ is the isotropy subgroup of the action (2.6).

Suppose $f$ is of degenerate type. Then the isotropy subgroup $G_{2}$ of the action (2.6) is $G_{1}$ itself, and $L_{1}$ is the bundle of Frenet frames. On $L_{1}, \Omega^{3}=\Omega_{l}^{3}=\Omega_{2}^{3}=0$. Consider the involutive left-invariant distribution on PGL $(4 ; \mathbf{R})$ defined by the exterior differential system $\left\{\Omega^{3}=\Omega_{l}^{3}=\Omega_{2}^{3}\right.$ $=0\}$. The analytic subgroup of $\operatorname{PGL}(4 ; \mathbf{R})$ corresponding to this distribution is

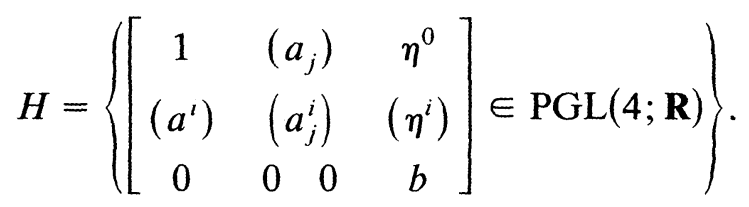

Hence $f(S) \subseteq H / H \cap G_{0} \cong \mathbf{R P}^{2}$.

We characterize the remaining (constant) type surfaces by the following theorem.

THEOREM 2.2. Let $\left(x^{1}, x^{2}, f(x)\right)$ be a surface in $\mathbf{R P}^{3}$, and let

$$
f_{i j}(x)=\frac{\partial^{2} f}{\partial x^{i} \partial x^{j}}(x) \text {. }
$$

Then

(i) if is of parabolic type if and only if $\operatorname{rank}\left(f_{i j}\right) \equiv 1$,

(ii) $f$ is of elliptic type if and only if $\operatorname{rank}\left(f_{i j}\right) \equiv 2$ and determinant $\left(f_{i j}\right)>0$,

(iii) if is of hyperbolic type if and only if $\operatorname{rank}\left(f_{i j}\right) \equiv 2$ and determinant $\left(f_{i j}\right)<0$.

The proof is computational. (See Theorem (2.11) of [8].) 
We now restrict our attention to the hyperbolic type surfaces. The analyses of other types are completely similar using the theory of moving frames. However the hyperbolic type surfaces admit perhaps simpler geometric interpretations due to the existence of asymptotic coordinates. Note that the hyperbolic type surfaces are "negatively curved".

From (2.6) the identity component of the isotropy group $G_{2}$ is computed

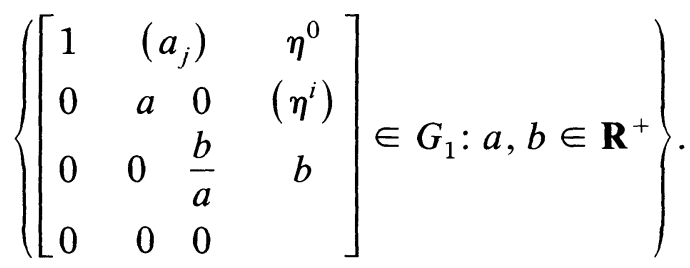

Let

$$
E_{6}=\left|\begin{array}{llll}
0 & 0 & 0 & 0 \\
0 & 0 & 0 & 0 \\
0 & 1 & 0 & 0 \\
0 & 0 & 0 & 0
\end{array}\right|, \quad E_{7}=\left|\begin{array}{cccc}
0 & 0 & 0 & 0 \\
0 & 0 & 1 & 0 \\
0 & 0 & 0 & 0 \\
0 & 0 & 0 & 0
\end{array}\right| \text { and } \quad E_{8}=\left|\begin{array}{cccc}
0 & 0 & 0 & 0 \\
0 & 0 & 0 & 0 \\
0 & 0 & 1 & 0 \\
0 & 0 & 0 & 0
\end{array}\right| .
$$

We also let $\mathscr{M}_{2}=\operatorname{span}\left\{E_{6}, E_{7}, E_{8}\right\}$. Then we have a vector space direct sum decomposition $\mathscr{G}_{1}=\mathscr{G}_{2} \oplus \mathscr{M}_{2}$, where $\mathscr{G}_{2}$ is the Lie algebra of $G_{2}$. The $\mathscr{M}_{2}$-component of $\Omega$ is given by

$$
\Omega_{1}^{2} \otimes E_{6} \oplus \Omega_{2}^{1} \otimes E_{7} \oplus\left(\Omega_{1}^{1}+\Omega_{2}^{2}-\Omega_{3}^{3}\right) \otimes E_{8} .
$$

Computing the action of $G_{2}$ on $\mathscr{M}_{2}$ we discover that there are four orbit types at the next stage. We list them below.

(2.9) Type IIIa (degenerate type): $\Omega_{1}^{2}-\Omega_{2}^{1}=\Omega_{1}^{1}+\Omega_{2}^{2}-\Omega_{3}^{3}=0$ on $L_{3}$. Type IIIb: $\Omega_{1}^{1}+\Omega_{2}^{2}-\Omega_{3}^{3}=0, \Omega_{2}^{1}=0, \Omega_{1}^{2}=\Omega^{1}$ on $L_{3}$.

Type IIIc: $\Omega_{1}^{1}+\Omega_{2}^{2}-\Omega_{3}^{3}=0, \Omega_{1}^{2}=0, \Omega_{2}^{1}=\Omega^{2}$ on $L_{3}$.

Type IIId (generic type): $\Omega_{1}^{1}+\Omega_{2}^{2}-\Omega_{3}^{3}=0, \Omega_{2}^{1}=\Omega^{2}, \Omega_{1}^{2}=\Omega^{1}$ on $L_{3}$.

The first three orbit types represent ruled surfaces. We restrict ourselves to the generic type IIId. $L_{3}$ is then an integral submanifold of the exterior differential system $\left\{\Omega_{l}^{1}+\Omega_{2}^{2}-\Omega_{3}^{3}=0, \Omega_{2}^{1}=\Omega^{2}, \Omega_{l}^{2}=\Omega^{1}\right\}$ on $L_{2}$, and it is $G_{3}$-principal bundle over $S$. Computations reveal that the identity component of the isotropy group $G_{3}$ is

$$
\left\{\left[\begin{array}{cccc}
1 & a_{1} & a_{2} & \eta^{0} \\
0 & 1 & 0 & a_{2} \\
0 & 0 & 1 & a_{1} \\
0 & 0 & 0 & 1
\end{array}\right] \in G_{2}: a_{1}, a_{2}, \eta^{0} \in \mathbf{R}\right\}
$$


Letting

$$
\begin{gathered}
E_{9}=\left|\begin{array}{rrrr}
0 & 1 & 0 & 0 \\
0 & 0 & 0 & 0 \\
0 & 0 & 0 & -1 \\
0 & 0 & 0 & 0
\end{array}\right|, E_{10}=\left|\begin{array}{rrrr}
0 & 0 & 1 & 0 \\
0 & 0 & 0 & -1 \\
0 & 0 & 0 & 0 \\
0 & 0 & 0 & 0
\end{array}\right|, \\
E_{11}=\left|\begin{array}{rrrr}
0 & 0 & 0 & 0 \\
0 & 1 & 0 & 0 \\
0 & 0 & -1 & 0 \\
0 & 0 & 0 & 0
\end{array}\right| \text { and } E_{12}=\left|\begin{array}{llll}
0 & 0 & 0 & 0 \\
0 & 0 & 0 & 0 \\
0 & 0 & 1 & 0 \\
0 & 0 & 0 & 1
\end{array}\right|,
\end{gathered}
$$

we obtain the vector space direct sum decomposition

$$
\mathscr{G}_{2}=\mathscr{G}_{3} \oplus \mathscr{M}_{3},
$$

where $\mathscr{G}_{3}$ is the Lie algebra of $G_{3}$ and $\mathscr{M}_{3}=\operatorname{span}\left\{E_{9}, E_{10}, E_{11}, E_{12}\right\}$.

The $\mathscr{M}_{3}$-component of $\Omega$ is given by

$$
\left(\Omega_{1}-\Omega_{3}^{2}\right) \otimes E_{9} \oplus\left(\Omega_{2}-\Omega_{3}^{1}\right) \otimes E_{10} \oplus \Omega_{l}^{1} \otimes E_{11} \oplus \Omega_{3}^{3} \otimes E_{12} .
$$

Computations show that there is a single orbit type at the next stage and that the action of $G_{3}$ on $\mathscr{M}_{3}$ allows us to make $\Omega_{1}-\Omega_{3}^{2}=\Omega_{2}-\Omega_{3}^{1}=0$. The isotropy group $G_{4}$ is the identity.

$L_{4}$ is the bundle of Frenet frames. Computations show that: On $L_{4}$ we have

$$
\left\{\begin{array}{l}
\Omega^{3}=0, \\
\Omega_{l}^{3}=\Omega^{2}, \quad \Omega_{2}^{3}=\Omega^{1}, \\
\Omega_{l}^{1}+\Omega_{2}^{2}-\Omega_{3}^{3}=0, \quad \Omega_{2}^{2}=\Omega^{2}, \Omega_{l}^{2}=\Omega^{1}, \\
\Omega_{1}-\Omega_{3}^{2}=0, \quad \Omega_{2}-\Omega_{3}^{1}=0, \\
\Omega_{l}^{1}=2 a \Omega^{1}+b \Omega^{2}, \quad \Omega_{2}^{2}=a \Omega^{1}+2 b \Omega^{2}, \\
\Omega_{3}=p \Omega^{1}+q \Omega^{2}, \\
\Omega_{1}+\Omega_{3}^{2}=2 q \Omega^{1}+r \Omega^{2}, \quad \Omega_{2}+\Omega_{3}^{1}=s \Omega^{1}+2 p \Omega^{2},
\end{array}\right.
$$

where $a, b, p, q, r, s$ are functions on $L_{4}$.

The functions $a, b, p, q, r, s$ are called (non-trivial) contact invariants.

At this juncture, a slightly different perspective may be helpful: Let $\Sigma$ denote the exterior system on $\operatorname{PGL}(4 ; \mathbf{R})$ (with the independence condition that $\Omega^{1} \wedge \Omega^{2} \neq 0$ ) represented by the equations in (2.11). Also suppose that the functions $a, b, p, q, r, s$ are so chosen that $\Sigma$ is closed. That is, the functions $a, b, p, q, r, s$ are compatible (but otherwise arbitrarily chosen). Counting the number of equations in $\Sigma$ we see that there 
exists a unique solution of the form $u: S \rightarrow \operatorname{PGL}(4 ; \mathbf{R})$. Define $f: S \rightarrow \mathbf{R P}^{3}$ by $f \equiv \pi \circ u$, where $\pi$ : $\operatorname{PGL}(4 ; \mathbf{R}) \rightarrow \mathbf{R} \mathbf{P}^{3}$ is the projection. Then $u$ is the Frenet frame along $f$ and all negatively curved non-ruled surfaces in $\mathbf{R P}^{3}$ arise in this way.

3. Induced projective structures. In this section we give a brief description of induced projective structures on submanifolds of $\mathbf{R P}^{n}$. For details and proofs pertaining to this section readers should consult sections three and four of [8].

In contrast to the case of Riemannian geometry, given a submanifold in $\mathbf{R P}^{n}$ there are in general not one but many distinct induced projective structures on it. Roughly speaking, a choice of normal framing dtermines an induced structure.

Let $f: S^{p} \rightarrow \mathbf{R P}^{n}$ be a $p$-dimensional submanifold given locally as a graph $\left(x^{i}\right) \mapsto\left(x^{i}, f^{a}(x)\right)$ as in $\S 2$. Recall that the bundle of first order moving frames of $f$ is $L_{1}=\left\{u_{0} \cdot G_{1}\right\}$. (See 2.1.)

Let $H$ be the subgroup of $G_{1}$ given by

$$
H=\left\{\left[\begin{array}{ccc}
1 & \left(a_{j}\right) & 0 \\
0 & \left(a_{j}^{i}\right) & 0 \\
0 & 0 & I_{n-p}
\end{array}\right]: \begin{array}{l}
\left(a_{j}^{i}\right) \in \mathrm{GL}(p ; \mathbf{R}) \\
{ }^{\mathrm{t}}\left(a_{j}\right) \in \mathbf{R}^{p}
\end{array}\right\}
$$

Also, let $N$ be the normal subgroup of $G_{1}$ given by

(3.2) $N=\left\{\left[\begin{array}{cc} & \left(\eta_{b}\right) \\ I_{p+1} & \left(\eta_{b}^{i}\right) \\ 0 & \left(b_{b}^{a}\right)\end{array}\right]: \begin{array}{l}{ }^{\mathrm{t}}\left(\eta_{b}\right) \in \mathbf{R}^{n-p}\left(b_{b}^{a}\right) \in \mathrm{GL}(n-p ; R) \\ \left(\eta_{b}^{i}\right) \in \mathbf{R}^{p \times(n-p)}\end{array}\right\}$.

Then $G_{1}=H \cdot N$, a semi-direct product. And we identify $G_{1} / N$ with $H$. Observe that $H$ is isomorphic to the projective isotropy group.

Consider the quotient space $L_{1} / N$. Because $N$ is a normal subgroup of $G_{1}$, it follows from the general theory of principal bundles that $L_{1} / N \rightarrow S$ is a principal $G_{1} / N=H$ bundle. Furthermore, since $H$ is isomorphic to the projective isotropy group of $\mathbf{R} \mathbf{P}^{p}$, this bundle $L_{1} / N \rightarrow S$ is a candidate for defining a projective structure on $S$, provided that there is some natural way to imbed the bundle $L_{1} / N \rightarrow S$ into $Q(S) \rightarrow S$, where $Q(S)$ is the principal bundle of quadratic frames over $\mathbf{R P}^{n}$. (I.e., the bundle of two-jets of local diffeomorphisms $\left(\mathbf{R}^{p}, 0\right) \rightarrow S$.)

We have the following proposition from [8]. 
Proposition 3.1. Any map $\eta: S \rightarrow N$ determines a natural bundle map section $i_{\eta}: L_{1} / N \rightarrow L_{1}$.

Roughly speaking, a map $\eta: S \rightarrow N$ corresponds to a normal frame field along $S$.

The following commutative diagram defines $I_{\eta}: L_{1} / N \rightarrow f^{-1} Q\left(\mathbf{R P}^{n}\right)$.

$$
\begin{array}{rcccc} 
& f^{-1} Q\left(\mathbf{R P}^{n}\right) & \rightarrow & Q\left(\mathbf{R P}^{n}\right) \\
& \stackrel{I_{\eta} \nearrow}{i_{\eta}} & \uparrow & & \uparrow \\
L_{1} / N \quad \stackrel{ }{\rightarrow} & L_{1} \subset L_{0} & \rightarrow & \operatorname{PGL}(n+1 ; \mathbf{R})
\end{array}
$$

The Maurer-Cartan form plays a crucial role.

Proposition 3.2.

(i) $\left.I_{\eta}^{*} \Theta^{\prime}\right|_{f^{-1} Q\left(\mathbf{R P}^{n}\right)}=\left.i_{\eta}^{*}\left(\Omega^{i}, \Omega_{j}^{i}\right)\right|_{L_{1}}$, where $\Theta^{\prime}=\left(\theta^{i}, \theta_{j}^{i}\right)=$ the $\mathbf{R}^{p} \oplus$ $\mathscr{G} \mathscr{L}(p ; \mathbf{R})$-component of the canonical form $\Theta$ of $Q\left(\mathbf{R P}^{n}\right)$, and

(ii) $\left.I_{\eta}^{*} \boldsymbol{\theta}^{a}\right|_{f^{-1} Q\left(\mathbf{R P}^{n}\right)}=0$, for $p+1 \leq a \leq n$.

For details of the properties of $Q(M)$ and $\Theta$, we refer to [7, Chapter VI, §5].

Definition 3.3. An induced projective structure on $f$ is a pair $\left(L_{1} / N, I_{\eta}\right)$.

(3.3) Strictly speaking, the induced projective structures do not depend on the $\left(\eta^{b}\right)$-part of the map $\eta: S \rightarrow N$ (using the notation in (3.2)). Let $\pi: L_{1} \rightarrow L\left(\mathbf{R P}^{n}\right)$ be the projection consistent with the forgetful functor $Q\left(\mathbf{R P}^{n}\right) \rightarrow L\left(\mathbf{R P}^{n}\right)$. (Note that $L_{1} \subset \operatorname{PGL}(n+1 ; \mathbf{R}) \subset Q\left(\mathbf{R P}^{n}\right)$.) Also let $\pi^{\prime}: G_{1} \rightarrow \mathrm{GL}(n: \mathbf{R})$ denote the group map of $\pi$. Then the totality of induced projective structures on $f$ is parametrized by the maps $S \rightarrow$ $\pi^{\prime}(N)$, i.e., by sections of the bundle $\pi\left(L_{1}\right) \rightarrow \pi\left(L_{1}\right) / \pi^{\prime}(N)$.

The next proposition states that an induced projective structure is indeed a projective structure. See [8] for a proof, and for the definition of projective structure.

Proposition 3.4. There is a naturally defined bundle imbedding $\Phi_{\eta}$ : $L_{1} / N \rightarrow Q(S)$ so that

$$
\Phi_{\eta}^{*} \Theta_{Q(S)}=\left.I_{\eta}^{*} \Theta^{\prime}\right|_{f^{-1} Q\left(\mathbf{R P}^{n}\right)},
$$

where $\Theta_{Q(S)}$ is the canonical form of $Q(S)$. 
The following lemma is technically important, (and is proved in [8]).

LEMMA 3.5. Any first order moving frame field $u: S \rightarrow L_{1}$ can be written as $u=i_{\eta} \circ q$ for some $\eta: S \rightarrow N$ and a section $q: S \rightarrow L_{1} / N$.

Let $f, \bar{f}: S^{p} \rightarrow \mathbf{R P}^{n}$ be two submanifolds and $\eta, \bar{\eta}: S \rightarrow N$ be maps as before.

Definition 3.6. We say that $f$ and $\bar{f}$ are (projectively) equivalent to each other with respect to the pair $(\eta, \bar{\eta})$ if the projective structures $\left(L_{1} / N, I_{\eta}\right)$ and $\left(\bar{L}_{1} / N, I_{\bar{\eta}}\right)$ are equivalent (as abstract projective structures, again see [8] for more details on equivalence).

(3.4) Suppose that $f$ and $\bar{f}$ are equivalent with respect to the pair $(\eta, \bar{\eta})$. Then given any section $q: S \rightarrow L_{1} / N$, there exists a corresponding section $\bar{q}: S \rightarrow \bar{L}_{1} / N$ defined by $\bar{q}=j \circ q$, where $J: L_{1} / N \rightarrow \bar{L}_{1} / N$ is the bundle isomorphism giving the equivalence. And by the definition of the equivalence of abstract projective structures we have

$$
\left.\left(i_{\eta} \circ q\right)^{*}\left(\Omega^{i}, \Omega_{j}^{i}\right)\right|_{L_{1}}=\left.\left(i_{\bar{\eta}} \circ \bar{q}\right)^{*}\left(\Omega^{i}, \Omega_{j}^{i}\right)\right|_{\bar{L}_{1}} .
$$

(3.5) Conversely, suppose that we are given $u=i_{\eta} \circ q: S \rightarrow L_{1}$ and $\bar{u}=i_{\bar{\eta}} \circ q: S \rightarrow \bar{L}_{1}$ such that $\left.u^{*}\left(\Omega^{i}, \Omega_{j}^{i}\right)\right|_{L_{1}}=\left.\bar{u}^{*}\left(\Omega^{i}, \Omega_{j}^{i}\right)\right|_{\bar{L}_{1}}$. Then it is not hard to see that there is a bundle isomorphism $J: L_{1} / N \rightarrow \bar{L}_{1} / N$ with $\bar{q}=J \circ q$ giving the equivalence between $\left(L_{1} / N, I_{\eta}\right)$ and $\left(\bar{L}_{1} / N, I_{\bar{\eta}}\right)$. (See [8], Lemma (4.3).)

In view of the discussion in this section we can ask several different questions regarding the equivalence of induced projective structures depending on the amount of restrictions placed upon projective frames. One of these questions, where there are no restrictions on projective frames, is dealt with in [8]. However, as we shall see, the notion of projective deformations considered by E. Cartan places a severe himitation upon the choice of projective frames.

4. Projective deformations of submanifolds of $\mathbf{R P}^{n}$. In this section we derive exterior differential systems describing projective deformations and draw some general conclusions.

Let $f, \bar{f}: S, S \rightarrow \mathbf{R P}^{n}$ be two $p$-dimensional submanifolds of $\mathbf{R P}^{n}$. 
DEFINITION 4.1. $f$ and $\bar{f}$ are $r$ th order projective deformations of each other if there exists a smooth map $v: S \rightarrow \operatorname{PGL}(n+1 ; \mathbf{R})$ and a diffeomorphism $F: S \rightarrow \bar{S}$ such that, for each point $s \in S, f$ and $v(s) \circ \bar{f} \circ F$ define the same $r$-jet at $s$.

Since $r$ th order deformation implies $r$ th order contact, for sufficiently large $r, r$ th order deformation problems admit only congruent solutions. In fact, if induced structures under consideration are $G$-structures of degree one then non-trivial first order deformations are already rare. (A somewhat incidental remark is that the notion of first order deformation is in general more "rigid" than the notion of equivalence of induced $G$-structres of degree one.)

However, in our case we have $G$-structures of degree two. That is to say, projective structures are naturally subbundles of the quadratic bundle. This will be reflected in Theorem 4.4.

Let $f, \bar{f}: S, \bar{S} \rightarrow \mathbf{R P}^{n}$ be $p$-dimensional submanifolds of $\mathbf{R} \mathbf{P}^{n}$ and let $L_{0}$ and $L_{1}$ (respectively $\bar{L}_{0}$ and $\bar{L}_{1}$ ) denote the bundles of zeroth and first order moving frames on $f$ (respectively on $\bar{f}$ ).

Some computations, using Proposition 2 of $\S 4$ in [6], give the following theorems.

THEOREM 4.2. Suppose $f$ and $\bar{f}$ are first order projective deformations of each other. Then there exist $u: S \rightarrow L_{1}, \bar{u}: S \rightarrow \bar{L}_{0}$ and $F: S \rightarrow \bar{S} a$ diffeomorphism such that $\left.u^{*}\left(\Omega^{i}\right)\right|_{L_{1}}=\left.(\bar{u} \circ F) *\left(\Omega^{i}\right)\right|_{\bar{L}_{0}}$. Conversely, suppose we have $(u, \bar{u}): S, \bar{S} \rightarrow \operatorname{PGL}(n+1 ; \mathbf{R})$ and $F: S \rightarrow \bar{S}$ a diffeomorphism so that $u^{*}\left(\Omega^{i}\right)=(\bar{u} \circ F)^{*}\left(\Omega^{i}\right)$. Then $f \equiv \pi \circ u$ and $\bar{f} \equiv \pi \circ u$ are first order deformations of each other, where $\pi: \operatorname{PGL}(n+1 ; \mathbf{R}) \rightarrow \mathbf{R P}^{n}$ is the natural projection given by $\pi(u)=u(0)$.

THEOREM 4.3. Suppose $f$ and $\bar{f}$ are second order projective deformations of each other. Then for any first order frame field $u: S \rightarrow L$, along $f$ there exists a first order frame field $\bar{u}: \bar{S} \rightarrow \bar{L}_{1}$ and $\bar{f}$, and a diffeomorphism $F$ : $S \rightarrow \bar{S}$ such that

$$
\omega^{i}=\bar{\omega}^{i}, \quad \omega_{j}^{i}=\bar{\omega}_{j}^{i}, \quad \omega_{j}^{a}=\bar{\omega}_{j}^{a}
$$

where $\omega^{i}=u^{*} \Omega^{2}$, etc. Conversely, if $u: S \rightarrow \operatorname{PGL}(n+1 ; \mathbf{R})$ and $\bar{u}: \bar{S} \rightarrow$ $\operatorname{PGL}(n+1 ; \mathbf{R})$, and $F: S \rightarrow \bar{S}$ is a diffeomorphism, such that $u$ and $\bar{u} \circ F$ satisfy (4.1) as well as

$$
u^{*} \Omega^{a}=0=(\bar{u} \circ F)^{*} \Omega^{a},
$$

then $f=\pi \circ u$ and $\bar{f}=\pi \circ \bar{u}$ are second order deformations of each other. 
REMARK. In the above, one can replace $\bar{u}$ by $\bar{u} \circ F$, and thus dispense with $F$ and $\bar{S}$.

COROLlaRY. If first order frames $u$ and $\bar{u}$ along $f$ and $\bar{f}$, respectively, satisfy (4.1), then they also satisfy

$$
\omega_{n}^{n}=\bar{\omega}_{n}^{n}
$$

Proof. This will follow from the calculations leading up to (4.8).

We now prove:

THEOREM 4.4. Any two real-analytic p-dimensional submanifolds in $\mathbf{R P}^{n}$ are first order deformations of each other.

Proof. Let $f, \bar{f}: S, \bar{S} \rightarrow \mathbf{R} \mathbf{P}^{n}$ be the given submanifolds and let $L_{1}$ and $\bar{L}_{1}$ denote the bundles of first order moving frames on $f$ and $\bar{f}$ respectively. We consider the exterior differential system on $L_{1} \times \bar{L}_{1}$ given by

$$
\Sigma:\left\{\left.\Omega^{i}\right|_{L_{1}}=\left.\Omega^{i}\right|_{L_{1}}, i=1,2, \ldots, p\right\}
$$

with specified independent variables $\left.\Omega^{1}\right|_{L_{1}},\left.\Omega^{2}\right|_{L_{1}}, \ldots,\left.\Omega^{p}\right|_{L_{1}}$. Writing the quadratic equations of $\Sigma$, modulo $\Sigma$, we obtain

$$
d \Sigma:\left\{\left.\left(\left.\Omega_{k}^{i}\right|_{L_{1}}-\left.\Omega_{k}^{i}\right|_{\bar{L}_{1}}\right) \wedge \Omega^{k}\right|_{L_{1}} \equiv 0, i=1,2, \ldots, p\right\} .
$$

The system is in normal form and applying Cartan's involutivity criterion the system is seen to be involutive.

The rest of the proof follows easily using Theorem 4.2.

We now ask the question: Given $f: S \rightarrow \mathbf{R P}^{n}$ do there exist non-trivial second order deformations of it?

We consider the exterior differential system on $L_{1} \times \operatorname{PGL}(n+1 ; \mathbf{R})$ with specified independent variables $\omega^{1}, \omega^{2}, \ldots, \omega^{p}$ given by

$$
\Sigma:\left\{\omega^{i}=\Omega^{i}, \omega_{j}^{i}=\Omega_{j}^{i}, \omega_{j}^{a}=\Omega_{j}^{a}, 1 \leq i, j \leq p, p+1 \leq a \leq n\right\},
$$

where $\omega^{i}=\Omega_{L_{1}}, \omega_{j}^{i}=\left.\Omega_{j}^{i}\right|_{L_{1}}$ and $\omega_{j}^{a}=\left.\Omega_{j}\right|_{L_{1}}$.

Note that here, and elsewhere, we write $\omega^{i}$ in place of $\pi^{*} \omega^{i}$, where $\pi$ : $L_{1} \times \operatorname{PGL}(n+1 ; \mathbf{R}) \rightarrow L_{1}$, etc. Hopefully this abuse of notation will not cause any confusion.

Suppose we have a $p$-dimensional solution

$$
(u, \bar{u}): S \rightarrow L_{1} \times \operatorname{PGL}(n+1 ; \mathbf{R})
$$


of the system $\Sigma$ in (4.1). Then $\bar{f}$ defined by $\bar{f} \equiv \pi \circ \bar{u}$ is a second order deformation of $f$ by Theorem 4.3.

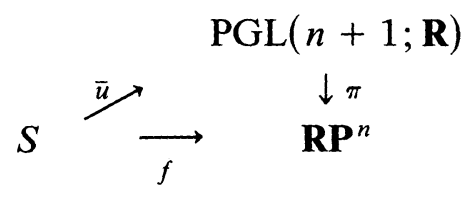

Moreover, it is easy to see that if $F: S \rightarrow \bar{S}$ is a diffeomorphism then $\bar{f} \circ F^{-1}: \bar{S} \rightarrow \mathbf{R P}^{n}$ is also a second order deformation of $f$.

We close the system $\Sigma$ and write the quadratic equations modulo the system.

$$
\left\{\begin{array}{l}
\Omega_{a}^{i} \wedge \Omega^{a} \equiv 0, \\
-\left(\omega_{a}^{i}-\Omega_{a}^{i}\right) \wedge \omega_{j}^{a}-\omega^{i} \wedge\left(\omega_{j}-\Omega_{j}\right)+\delta_{j}^{i}\left(\omega_{k}-\Omega_{k}\right) \wedge \omega^{k} \\
\left(\omega_{b}^{a}-\Omega_{b}^{a}\right) \wedge \omega_{j}^{b}-\Omega^{a} \wedge \Omega_{j} \equiv 0 .
\end{array}\right.
$$

The above system is not in normal form and the general analysis is complicated. Suffice it to say that the system is over-determined and the prolongation of the system is difficult. Instead of the system (4.3) we consider a new system by imposing a genericity condition on solutions and by abusing the notation we call it $\Sigma$ once again.

$$
\begin{aligned}
\Sigma:\left\{\omega^{i}=\Omega^{i}, \omega_{j}^{i}=\Omega_{j}^{i}, \omega_{j}^{a}=\Omega_{j}^{a}, \Omega^{a}\right. & =0, \\
1 & \leq i, j \leq p, p+1 \leq a \leq n\} .
\end{aligned}
$$

The system is on $L_{1} \times \operatorname{PGL}(n+1 ; \mathbf{R})$ and has designated independent variables $\omega^{1}, \omega^{2}, \ldots, \omega^{p}$.

We compute the quadratic equations of the system, modulo the system as usual, and obtain

$$
\left\{\begin{array}{l}
-\left(\omega_{a}^{i}-\Omega_{a}^{i}\right) \wedge \omega_{j}^{a}-\omega^{i} \wedge\left(\omega_{j}-\Omega_{j}\right)+\delta_{j}^{i}\left(\omega_{k}-\Omega_{k}\right) \wedge \omega^{k} \equiv 0 \\
\left(\omega_{b}^{a}-\Omega_{b}^{a}\right) \wedge \omega_{j}^{b} \equiv 0
\end{array}\right.
$$

The above system is in homogeneous normal form with unknowns

$$
\left(\omega_{a}^{i}-\Omega_{a}^{i}\right), \quad\left(\omega_{j}-\Omega_{j}\right) \text { and }\left(\omega_{b}^{a}-\Omega_{b}^{a}\right)
$$

Write

$$
\Pi_{a}^{l}=\omega_{a}^{l}-\Omega_{a}^{i}, \quad \Pi_{\jmath}=\omega_{j}-\Omega_{j}, \quad \Pi_{b}^{a}=\omega_{b}^{a}-\Omega_{b}^{a} .
$$


Then (4.5) becomes

$$
\left\{\begin{array}{l}
\left(-A_{j k}^{a} \Pi_{a}^{i}+\delta_{k}^{i} \Pi_{j}+\delta_{j}^{i} \Pi_{k}\right) \wedge \omega^{k} \equiv 0 \\
A_{j k}^{b} \Pi_{b}^{a} \wedge \omega^{k} \equiv 0
\end{array}\right.
$$

Recall that $\omega_{j}^{a}=A_{j k}^{a} \omega^{k}$ on $L_{1}$. (See (2.4).)

We now restrict ourselves to hypersurfaces in $\mathbf{R} \mathbf{P}^{n},(4.6)$ becomes

$$
\left\{\begin{array}{l}
\left(-A_{j k} \Pi_{n}^{i}+\delta_{k}^{i} \Pi_{j}+\delta_{j}^{i} \Pi_{k}\right) \wedge \omega^{k} \equiv 0 . \\
A_{j k} \Pi_{n}^{n} \wedge \omega^{k} \equiv 0 .
\end{array}\right.
$$

We assume that rank $A_{j k} \geq 2$. Note that this corresponds to either elliptic or hyperbolic type surfaces in $\mathbf{R P}^{3}$. By Cartan's lemma it follows that $\Pi_{n}^{n}=0$. We now have a partially prolonged system given by

$$
\left\{\omega^{i}=\Omega^{i}, \omega_{j}^{i}=\Omega_{j}^{i}, \omega_{j}^{n}=\Omega_{j}^{n}, \Omega^{n}=0, \omega_{n}^{n}=\Omega_{n}^{n},\right.
$$

$$
1 \leq i, j \leq p=n-1\} \text {. }
$$

Exterior differentiations give

$$
\left\{\begin{array}{l}
\left(-A_{j k} \Pi_{n}^{i}+\delta_{k}^{i} \Pi_{j}+\delta_{j}^{i} \Pi_{k}\right) \wedge \omega^{k} \equiv 0 \\
A_{j k} \Pi_{n}^{i} \wedge \omega^{k} \equiv 0
\end{array}\right.
$$

The system is once again over-determined.

5. Surfaces in $\mathbf{R P} \mathbf{P}^{3}$. Having the remark at the end of $\S 3$ in mind we make the following definition.

Definition 5.1. Let $f, \bar{f}: S^{p} \rightarrow \mathbf{R P}^{n}$ be two submanifolds. Also let $u=i_{\eta} \circ q: S \rightarrow L_{1}$ and $\bar{u}=i_{\bar{\eta}} \circ \bar{q}: S \rightarrow \bar{L}_{1}$ be projective frames along $f$ and $\bar{f}$ respectively. Then we say that $f$ and $\tilde{f}$ are projectively equivalent to each other with respect to the pair $(u, \bar{u})$ if the induced projective structures $\left(L_{1} / N, I_{\eta}\right)$ and $\left(\bar{L}_{1} / N, \bar{I}_{\bar{\eta}}\right)$ are equivalent and the resulting equivalence carries $q$ to $\bar{q}$.

The following lemma is merely a reformulation of the above definition.

LEMMA 5.2. Keeping the same notation as in the above definition, $f$ and $\bar{f}$ are projectively equivalent with respect to the pair $(u, \bar{u})$ if and only if $\left.u^{*}\left(\Omega^{i}, \Omega_{j}^{i}\right)\right|_{L_{1}}=\left.\bar{u}^{*}\left(\Omega^{i},: \Omega_{j}^{i}\right)\right|_{\bar{L}_{1}}$

We now make a detailed study of second order deformations of surfaces in $\mathbf{R P}^{3}$. To do so, we recall earlier notations from $\$ 4$ and restrict ourselves to hyperbolic type surfaces in $\mathbf{R P}^{3}$. (So $p=2$ and $n=3$.) 
The system (4.8) becomes

$$
\Sigma:\left\{\omega^{i}=\Omega^{i}, \Omega^{3}=0, \omega_{j}^{i}=\Omega_{j}^{i}, \Omega_{j}^{3}=\Omega_{j}^{3}, \omega_{3}^{3}=\Omega_{3}^{3}, i, j=1,2\right\} .
$$

The system is on $L_{1} \times \operatorname{PGL}(4 ; \mathbf{R})$ with specified independent variables $\omega^{1}$ and $\omega^{2}$.

Observe that from the computations we made in $\$ 2$ (see (2.9)) it follows that the solutions $\bar{f}=\pi \circ \bar{u}$ of the above system have the same third order type as $f$. In other words, if we assume that $f$ has constant third order type IIId (the generic type), then any second order projective deformation of $f$ is also of generic type. Thus assuming $f$ to be of generic type, without loss of generality we restrict our system to $L_{3} \times \operatorname{PGL}(4 ; \mathbf{R})$. We take the generic type IIId.

We write down the quadratic equations of $\Sigma$.

$$
\left\{\begin{array}{l}
{\left[\left(\omega_{3}^{1}-\omega_{2}\right)-\left(\Omega_{3}^{1}-\Omega_{2}\right)\right] \wedge \omega^{2}+2 \omega^{1} \wedge\left(\omega_{1}-\Omega_{1}\right) \equiv 0} \\
{\left[\left(\omega_{3}^{1}-\omega_{2}\right)-\left(\Omega_{3}^{1}-\Omega_{2}\right)\right] \wedge \omega^{1} \equiv 0} \\
{\left[\left(\omega_{3}^{2}-\omega_{1}\right)-\left(\Omega_{3}^{2}-\Omega_{1}\right)\right] \wedge \omega^{2} \equiv 0} \\
{\left[\left(\omega_{3}^{2}-\omega_{1}\right)-\left(\Omega_{3}^{2}-\Omega_{1}\right)\right] \wedge \omega^{1}+2 \omega^{2} \wedge\left(\omega_{2}-\Omega_{2}\right) \equiv 0} \\
\theta^{1} \wedge\left[\left(\omega_{3}^{2}+\omega_{1}\right)-\left(\Omega_{3}^{2}+\Omega^{1}\right)\right] \\
\quad+\theta^{2} \wedge\left[\left(\omega_{3}^{1}+\omega_{2}\right)-\left(\Omega_{3}^{1}+\Omega_{2}\right)\right] \equiv 0
\end{array}\right.
$$

Letting $\Pi^{1}=\left(\omega_{3}^{1}-\omega_{2}\right)-\left(\Omega_{3}^{1}-\Omega_{2}\right), \Pi^{2}=\left(\omega_{3}^{2}-\omega_{1}\right)-\left(\Omega_{3}^{2}-\Omega_{1}\right), \Pi^{3}$ $=2\left(\omega_{1}-\Omega_{1}\right)$ and $\Pi^{4}=2\left(\omega_{2}-\Omega_{2}\right)$, we rewrite $(5.2)$.

$$
\left\{\begin{array}{rlr}
\Pi^{1} \wedge \omega^{2} & -\Pi^{3} \wedge \omega^{1} & \equiv 0 \\
\Pi^{1} \wedge \omega^{1} & & \equiv 0 \\
& \Pi^{2} \wedge \omega^{2} & \equiv 0 \\
\Pi^{2} \wedge \omega^{1} & -\Pi^{4} \wedge \omega^{2} & \equiv 0 \\
\Pi^{1} \wedge \omega^{2}+\Pi^{2} \wedge \omega^{1}+\Pi^{3} \wedge \omega^{1}+\Pi^{4} \wedge \omega^{2} & \equiv 0 .
\end{array}\right.
$$

We have five equations and four unknowns and the system is overdetermined. According to the general theory the system requires at the most four successive prolongations.

We prolong the system by setting $\Pi^{a}=l_{j}^{a} \omega^{j}$, where $1 \leq a \leq 4$. Substituting into (5.3) we obtain

$$
\left\{\begin{array}{l}
l_{1}^{1}+l_{2}^{3}=0 \\
l_{2}^{1}=0 \\
l_{1}^{2}=0 \\
l_{2}^{2}+l_{1}^{4}=0 \\
l_{1}^{1}-l_{2}^{2}-l_{2}^{3}+l_{1}^{4}=0 .
\end{array}\right.
$$


We obtain three dimensional solutions given by $l_{2}^{1}=l_{1}^{2}=0$, and $l_{1}^{1}=l_{2}^{2}=-l_{2}^{3}=-l_{1}^{4}, l_{1}^{3}, l_{2}^{4}$ arbitrary.

The prolonged system is on $L_{3} \times \operatorname{PGL}(4 ; \mathbf{R}) \times \mathbf{R}^{3}$.

$$
\begin{aligned}
\hat{\Sigma}^{\prime}=\Sigma \cup\left\{\Pi^{1}=l_{1}^{1} \omega^{1}, \Pi^{2}=l_{1}^{1} \omega^{2}, \Pi^{3}=l_{1}^{3} \omega^{1}-l_{1}^{1} \omega^{2},\right. \\
\left.\Pi^{4}=-l_{1}^{1} \omega^{1}+l_{2}^{4} \omega^{2}\right\} .
\end{aligned}
$$

We now have four equations and three unknowns.

Suppose $(u, \bar{u}): S \rightarrow L_{3} \times \operatorname{PGL}(4 ; \mathbf{R})$ solves the above system. Then in particular we must have

$$
\left\{\begin{array}{l}
(u, \bar{u})^{*} \Pi^{1}=u^{*}\left(\omega_{3}^{1}-\omega_{2}\right)-\bar{u}^{*}\left(\Omega^{31}-\Omega_{2}\right)=t u^{*} \omega^{1} \\
(u, \bar{u})^{*} \Pi^{2}=u^{*}\left(\omega_{3}^{2}-\omega_{1}\right)-\bar{u}^{*}\left(\Omega_{3}^{2}-\Omega_{1}\right)=t u^{*} \omega^{2}
\end{array}\right.
$$

for some function $t$ on $S$. Let

$$
K=\left[\begin{array}{cccc}
1 & 0 & 0 & t / 2 \\
0 & 1 & 0 & 0 \\
0 & 0 & 1 & 0 \\
0 & 0 & 0 & 1
\end{array}\right]: S \rightarrow G_{3}
$$

(recall from (2.9) that $G_{3}$ is the isotropy group for $L_{3}$ ). Also let $\hat{u}=u K$. Observe that $(\hat{u}, \bar{u})$ also solves the system with $t \equiv 0$. Hence, without losing generality, we replace (5.5) by

$$
\Sigma \cup\left\{\Pi^{1}=0, \Pi^{2}=0, \Pi^{3}=l_{1}^{3} \omega^{1}, \Pi^{4}=l_{2}^{4} \omega^{2}\right\} .
$$

Differentiate both sides of the equations $\Pi^{1}=0$ and $\Pi^{2}=0$ and obtain

$$
\left\{\begin{array}{l}
\omega^{2} \wedge\left(\omega_{3}-\Omega_{3}-l_{2}^{4} \omega^{1}\right) \equiv 0 \\
\omega^{1} \wedge\left(\omega_{3}-\Omega_{3}-l_{1}^{3} \omega^{2}\right) \equiv 0
\end{array}\right.
$$

It follows that $\omega_{3}-\Omega_{3}=l_{2}^{4} \omega^{1}+l_{1}^{3} \omega^{2}$.

The system is now on $L_{3} \times \operatorname{PGL}(4 ; \mathbf{R}) \times \mathbf{R}^{2}$.

$$
\begin{aligned}
& \Sigma \cup\left\{\Pi_{1}=0, \Pi_{2}=0\right\} \\
& \quad \cup\left\{\omega_{3}-\Omega_{3}=l_{2}^{4} \omega^{1}+l_{1}^{3} \omega^{2}, \Pi^{3}=l_{1}^{3} \omega^{1}, \Pi^{4}=l_{2}^{4} \omega^{2}\right\} .
\end{aligned}
$$

Recalling the computations we made in $\$ 2$ (see (2.11)) we see that the solutions $\bar{f}=\pi \circ \bar{u}$ of the above system are of the same fourth order type as $f$. We restrict the system to $L_{4} \times \operatorname{PGL}(4 ; \mathbf{R}) \times \mathbf{R}^{2}$. The isotropy subgroup $G_{4}$ is trivial and the Frenet frame field $u: S \rightarrow L_{4}$ defines a unique map $\eta: S \rightarrow N \triangleleft G_{1}$. Similarly if $\bar{u}: S \rightarrow \operatorname{PGL}(4 ; \mathbf{R})$ gives rise to a solution $\bar{f}$ then $\bar{u}$ actually is the Frenet frame field along $\bar{f}$ and it defines a unique map $\bar{\eta}: S \rightarrow N \triangleleft G_{1}$. Let $u=i_{\eta} \circ q$ and $\bar{u}=i_{\bar{\eta}} \circ \bar{q}$ be the decompositions given by Lemma 3.5 . 
We then have the following commutative diagram.

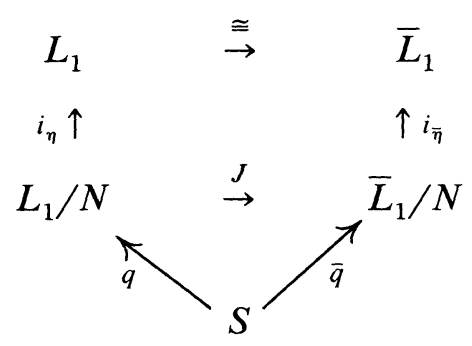

The top horizontal arrow is given by $u \mapsto v \cdot u$ where $v: S \rightarrow \operatorname{PGL}(4 ; \mathbf{R})$ achieves the deformations as in Definition 4.1. The middle horizontal arrow gives the projective equivalence with $\bar{q}=J \circ q$ as in (3.5).

THEOREM 5.3. Let $f, \bar{f}: S \rightarrow \mathbf{R P}^{3}$ be two non-ruled negatively curved surfaces in $\mathbf{R P}^{3}$. Then $f$ and $\bar{f}$ are second order deformations of each other if and only if $f$ and $\bar{f}$ are projectively equivalent with respect to the Frenet frames.

Proof. Suppose $f$ and $\bar{f}$ are second order deformations of each other. Let $u$ be the Frenet frame field along $f$. Then $(u, \bar{u})$ satisfy (4.1) and (4.2), (for $n=3$ ), for some first order frame field $\bar{u}$ along $\bar{f}$, by Theorem 4.3 and its Corollary. Recall that a Frenet frame is a fourth order frame, which is characterized by the table,

\begin{tabular}{l|l} 
First order & $\omega^{3}=0$ \\
Second order & $\omega_{l}^{3}=\omega^{2}, \omega_{2}^{3}=\omega^{1}$ \\
Third order & $\omega_{l}^{1}+\omega_{2}^{2}=\omega_{3}^{3}, \omega_{2}^{1}=\omega^{2}, \omega_{1}^{2}=\omega^{1}$ \\
Fourth order & $\omega_{1}=\omega_{3}^{2}, \omega_{2}=\omega_{3}^{1}$
\end{tabular}

where each order includes all the characterizations of the preceding orders as well. It follows then from (4.1) and (4.2), and as $u$ is the Frenet frame, that $\bar{u}$ is a third order frame field along $\bar{f}$.

By the calculations leading up to (5.6) we have

$$
\left\{\begin{array}{l}
\omega_{3}^{1}-\omega_{2}-\left(\bar{\omega}_{3}^{1}-\bar{\omega}_{2}\right)=t \omega^{1} \\
\omega_{3}^{2}-\omega_{1}-\left(\bar{\omega}_{3}^{2}-\bar{\omega}_{1}\right)=t \omega^{2}
\end{array}\right.
$$

for some function $t$ on $S$. Let

$$
\left[\begin{array}{cccc}
1 & 0 & 0 & t / 2 \\
0 & 1 & 0 & 0 \\
0 & 0 & 1 & 0 \\
0 & 0 & 0 & 1
\end{array}\right]: S \rightarrow G_{3}
$$


Then $\hat{u}: \bar{u} K$ is also a third order frame field along $\bar{f}$. A direct calculation shows that $(u, \hat{u})$ satisfy $(4.1),(4.2)$ and $(*)$ with $t=0$. Of course, in our case $\omega_{3}^{1}=\omega_{2}$ and $\omega_{3}^{2}=\omega_{1}$ as $u$ is the Frenet frame. Consequently $\hat{u}$ is the Frenet frame along $\bar{f}$. Then by Lemma 5.2, $f$ and $\bar{f}$ are projectively equivalent with respect to the pair of Frenet frames $(u, \hat{u})$. To prove the converse, we assume that $f$ and $\bar{f}$ are projectively equivalent with respect to the pair $(u, \bar{u})$ where once again $u, \bar{u}$ denote the Frenet frames along $f$ and $\bar{f}$ respectively. Then we have

$$
\left.u^{*}\left(\Omega^{i}, \Omega_{j}^{i}\right)\right|_{L_{1}}=\left.\bar{u}^{*}\left(\Omega^{i}, \Omega_{j}^{i}\right)\right|_{\bar{L}_{1}} .
$$

Now $u, \bar{u}$ are the Frenet frames. Thus

$$
u^{*} \Omega_{1}^{3}=u^{*} \Omega^{2}=\bar{u}^{*} \Omega^{2}=\bar{u}^{*} \Omega_{1}^{3} \quad \text { and } \quad u^{*} \Omega_{2}^{3}=u^{*} \Omega^{1}=\bar{u}^{*} \Omega^{1}=\bar{u}^{*} \Omega_{2}^{3} .
$$

That is, $(u, \bar{u})$ solves the system

$$
\begin{aligned}
\Sigma:\left\{\left.\Omega^{i}\right|_{L_{1}}=\left.\Omega^{i}\right|_{\bar{L}_{1}},\left.\Omega_{j}^{i}\right|_{L_{1}}=\left.\Omega_{j}^{i}\right|_{\bar{L}_{1}},\left.\Omega_{j}^{3}\right|_{L_{1}}=\left.\Omega_{j}^{3}\right|_{\bar{L}_{1}}, i, j=1,2\right\} & \text { on } L_{1} \times \bar{L}_{1} .
\end{aligned}
$$

Here, the author is thankful to the referee for suggesting a revision of the proof of Theorem 5.3 which made the proof more readable.

We continue our analysis of the system (5.7). We compute the quadratic equations, modulo the system as usual, and obtain

$$
\left\{\begin{array}{l}
\omega^{1} \wedge\left(d l_{1}^{3}-2 l_{1}^{3} \Omega_{1}^{1}\right) \equiv 0 \\
\omega^{2} \wedge\left(d l_{2}^{4}-2 l_{2}^{4} \Omega_{2}^{2}\right) \equiv 0 \\
\omega^{2} \wedge\left(d l_{1}^{3}-2 l_{1}^{3} \Omega_{1}^{1}\right)+\omega \wedge\left(d l_{2}^{4}-2 l_{2}^{4} \Omega_{2}^{2}\right) \equiv 0 .
\end{array}\right.
$$

We have three equations and two unknowns $d l_{1}^{3}$ and $d l_{2}^{4}$. The system is over-determined. We prolong the system by adding equations

$$
\left\{\begin{array}{l}
d l_{1}^{3}-2 l_{1}^{3} \Omega_{1}^{1}=l \omega^{1} \\
d l_{2}^{4}-2 l_{2}^{4} \Omega_{2}^{2}=l \omega^{2} .
\end{array}\right.
$$

Exterior differentiations of equations in (5.9) give

$$
\left\{\begin{array}{l}
d l \wedge \omega^{1}=-l \omega^{1} \wedge 3 \omega_{1}^{1}+l_{1}^{3} \omega^{1} \wedge\left(4 \omega_{1}-2 \omega^{2}\right), \\
d l \wedge \omega^{2}=-l \omega^{2} \wedge 3 \omega_{2}^{2}+2 l_{2}^{4} \omega^{2} \wedge\left(\omega_{2}^{2}-\omega^{1}\right) .
\end{array}\right.
$$

We have two equations and 1 unknown $d l$. We prolong the system by throwing in the equation

$$
d l=2 l_{2}^{4} A \omega^{1}+2 l_{1}^{3} B \omega^{2}-l \omega_{3}^{3},
$$


where

$$
A=\omega^{2} \wedge\left(2 \omega_{2}-\omega^{1}\right)\left(e_{1}, e_{2}\right), \quad B=\omega^{1} \wedge\left(\omega^{2}-2 \omega_{1}\right)\left(e_{1}, e_{2}\right),
$$

and $e_{1}, e_{2}$ are the frame field dual to $\omega^{1}, \omega^{2}$. Observe that $A$ and $B$ are known functions on $S$.

Differentiating both sides of the equation (5.11) we finally obtain the compatibility condition of E. Cartan

$$
2 l_{1}^{3}\left(d r \wedge \omega^{2}\right)+2 l_{2}^{4}\left(d s \wedge \omega^{1}\right)+3 l(r-s) \omega^{1} \wedge \omega^{2}=0,
$$

where $r$ and $s$ are contact invariants computed in (2.11).

If $r \equiv s \equiv$ constant then the equation (5.12) is identically satisfied. In this case a three parameter family of solutions is possible. Otherwise, we let $d r=r_{1} \omega^{1}+r_{2} \omega^{2}$ and $d s=s_{1} \omega^{1}+s_{2} \omega^{2}$. Then (5.12) becomes

$$
2 l_{1}^{3} r_{1}-2 l_{2}^{4} s_{2}+3 l(r-s)=0 .
$$

Solving for $l_{1}^{3}, l_{2}^{4}$ and $l$ we obtain at the most a two parameter family of solutions. This gives the

THEOREM 5.4. For a generic non-ruled negatively curved surface in $\mathbf{R P}^{3}$ there exists at the most a two parameter family of non-trivial second order deformations of it.

Settling the actual existence question of the above two parameter family of solutions would require a considerable amount of computations yet. A possible line of attack would be to set up an exterior system anew describing the system of differential equations coming from the compatibility condition.

\section{REFERENCES}

[1] R. Bryant, S. S. Chern and P. A. Griffiths, Notes on exterior differential systems, Proc. 1980 Peking Symposium on Differential Equations, Peking University, 1980.

[2] E. Cartan, Sur la déformation projective des surfaces, Ann. Ec. Norm., t. 37 (1920), 259-356.

[3] _ Les systèmes Differentiels Extérieurs et Leurs Applications Gémétriques, Hermann, Paris, 1945.

[4] Sur la connexion projective des surface, Vol. I, Oeuvres Complètes, (1955), 915-918.

[5] G. Jensen, Higher Order Contact of Submanifolds of Homogeneous Spaces, Lecture Notes in Math. 610, Springer-Verlag, Berlin, 1977.

[6] _ Deformation of submanifolds of homogeneous spaces, J. Differential Geom., 16 (1981), 213-246. 
[7] S. Kobayashi, Transformation Groups in Differential Geometry, Ergebrisse der Mathematik und ihrer Grenzebiete (70), Springer-Verlag, Berlin, 1972.

[8] K. Yang, Equivalence problems in projective differential geometry, Trans. Amer. Math. Soc., 282 (1984), 319-334.

Received January 10, 1984 and in revised form May 29, 1984. Research supported in part by NSF Epscor grant.

ARKansas State UnIVERSITY

STATE UNIVERSITY, AR 72467-0070 


\section{PACIFIC JOURNAL OF MATHEMATICS EDITORS}

\author{
V. S. VARADARAJAN (Managing Editor) \\ University of California \\ Los Angeles, CA 90024 \\ Charles R. DePrima \\ California Institute of Technology \\ Pasadena, CA 91125 \\ R. FINN \\ Stanford University \\ Stanford, CA 94305
}

\author{
HeRmanN FlaschKa \\ University of Arizona \\ Tucson, AZ 857.21
}

RAMESH A. GANGOLli

University of Washington

Seattle, WA 98195

ROBION KIRBY

University of California

Berkeley, CA 94720
C. C. MOORE

University of California

Berkeley, CA 94720

H. SAMELSON

Stanford University

Stanford, CA 94305

HAROLD STARK

University of California, San Diego

La Jolla, CA 92093

\section{ASSOCIATE EDITORS}
R. ARENS
E. F. BECKENBACH
B. H. NeumanN
F. WOLF
K. YoSHIDA
(1906-1982)

\section{SUPPORTING INSTITUTIONS}

\begin{abstract}
UNIVERSITY OF ARIZONA
UNIVERSITY OF BRITISH COLUMBIA

CALIFORNIA INSTITUTE OF TECHNOLOGY

UNIVERSITY OF CALIFORNIA

MONTANA STATE UNIVERSITY

UNIVERSITY OF NEVADA, RENO

NEW MEXICO STATE UNIVERSITY

OREGON STATE UNIVERSITY
\end{abstract}

\author{
UNIVERSITY OF OREGON \\ UNIVERSITY OF SOUTHERN CALIFORNIA \\ STANFORD UNIVERSITY \\ UNIVERSITY OF HAWAII \\ UNIVERSITY OF TOKYO \\ UNIVERSITY OF UTAH \\ WASHINGTON STATE UNIVERSITY \\ UNIVERSITY OF WASHINGTON
}

The Supporting Institutions listed above contribute to the cost of publication of this Journal, but they are not owners or publishers and have no responsibility for its content or policies.

Mathematical papers intended for publication in the Pacific Journal of Mathematics should be in typed form or offset-reproduced (not dittoed), double spaced with large margins. Please do not use built up fractions in the text of the manuscript. However, you may use them in the displayed equations. Underline Greek letters in red, German in green, and script in blue. The first paragraph must be capable of being used separately as a synopsis of the entire paper. In particular it should contain no bibliographic references. Please propose a heading for the odd numbered pages of less than 35 characters. Manuscripts, in triplicate, may be sent to any one of the editors. Please classify according to the scheme of Math. Reviews, Index to Vol. 39. Supply name and address of author to whom proofs should be sent. All other communications should be addressed to the managing editor, or Elaine Barth, University of California, Los Angeles, California 90024.

There are page-charges associated with articles appearing in the Pacific Journal of Mathematics. These charges are expected to be paid by the author's University, Government Agency or Company. If the author or authors do not have access to such Institutional support these charges are waived. Single authors will receive 50 free reprints; joint authors will receive a total of 100 free reprints. Additional copies may be obtained at cost in multiples of 50 .

The Pacific Journal of Mathematics is issued monthly as of January 1966. Regular subscription rate: $\$ 190.00$ a year (5 Vols., 10 issues). Special rate: $\$ 66.00$ a year to individual members of supporting institutions.

Subscriptions, orders for numbers issued in the last three calendar years, and changes of address should be sent to Pacific Journal of Mathematics, P.O. Box 969, Carmel Valley, CA 93924, U.S.A. Old back numbers obtainable from Kraus Periodicals Co., Route 100, Millwood, NY 10546.

The Pacific Journal of Mathematics at P.O. Box 969, Carmel Valley, CA 93924 (ISSN 0030-8730) publishes 5 volumes per year. Application to mail at Second-class postage rates is pending at Carmel Valley, California, and additional mailing offices. Postmaster: Send address changes to Pacific Journal of Mathematics, P.O. Box 969, Carmel Valley, CA 93924.

PUBLISHED BY PACIFIC JOURNAL OF MATHEMATICS, A NON-PROFIT CORPORATION

Copyright $\odot 1985$ by Pacific Journal of Mathematics 


\section{Pacific Journal of Mathematics}

Vol. 120, No. $2 \quad$ October, 1985

Philip Marshall Anselone and Mike Treuden, Regular operator

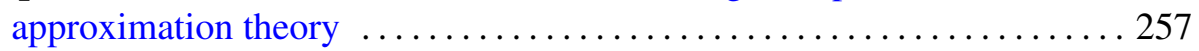

Giuseppe Baccella, Semiprime $\aleph-Q F 3$ rings $\ldots \ldots \ldots \ldots \ldots \ldots \ldots \ldots . \ldots \ldots$

Earl Robert Berkson and Thomas Alastair Gillespie, The generalized M.

Riesz theorem and transference $\ldots \ldots \ldots \ldots \ldots \ldots \ldots \ldots . \ldots 279$

Joachim Boidol, A Galois-correspondence for general locally compact

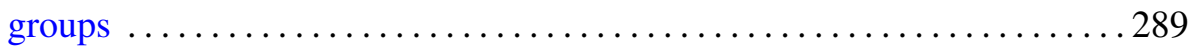

Joseph Eugene D'Atri, Josef Dorfmeister and Yan Da Zhao, The isotropy

representation for homogeneous Siegel domains ............... 295

C. Debiève, On Banach spaces having a Radon-Nikodým dual

Michael Aaron Freedman, Existence of strong solutions to singular

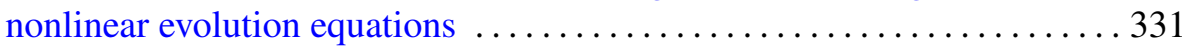

Francisco Jose Freniche, Grothendieck locally convex spaces of continuous

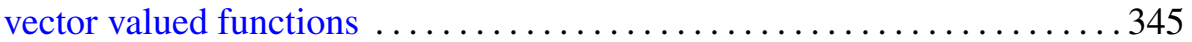

Hans-Peter Künzi and Peter Fletcher, Extension properties induced by complete quasi-uniformities . ............................ 357

Takaŝi Kusano, Charles Andrew Swanson and Hiroyuki Usami, Pairs of

positive solutions of quasilinear elliptic equations in exterior domains . . 385

Angel Rafael Larotonda and Ignacio Zalduendo, Spectral sets as Banach

manifolds

J. Martínez-Maurica and C. Pérez García, A new approach to the

Kreı̆n-Milman theorem

Christian Pommerenke, On the boundary continuity of conformal maps . . . 423

M. V. Subba Rao, Some Rogers-Ramanujan type partition theorems

Stephen Edwin Wilson, Bicontactual regular maps .........

Jaap C. S. P. van der Woude, Characterizations of (H)PI extensions

Kichoon Yang, Deformation of submanifolds of real projective space

Subhashis Nag, Errata: "On the holomorphy of maps from a complex to a

real manifold" 